silogística sólida puesta al servicio, naturalmente, de la demostración de las verdades de la fe, entre ellas los dogmas de la Trinidad y la Encarnación. Así, por ejemplo, el Liber de conuenientia se centra en demostrar la absoluta armonía existente entre creer (fides) y entender (intellectus), un hecho que implica no sólo la posibilidad, sino la obligación que todo intelectual cristiano tiene de probar racionalmente los fundamentos del credo cristiano. Domínguez (pp. 77-83) observa que se trata de un texto que conecta en muchos aspectos con la epístola que cierra el Liber de experientia realitatis Artis ipsius generalis (op. 138), en donde Llull afirma que los teólogos cristianos que defienden la imposibilidad de demostrar racionalmente los artículos de la fe lo hacen porque utilizan únicamente dos formas aristotélicas de demostración, per quia y propter quid, frente a lo cual el sabio mallorquín propone dos tipos de argumentos alternativos, la demonstratio per aequiparantiam y la demonstratio per hypothesim, que son precisamente empleados en la redacción del Liber de conuenientia. Merece destacarse el riguroso trabajo llevado a cabo por Domínguez para la fijación del texto de esta obra, caracterizada desde el punto de vista ecdótico por una transmisión ciertamente compleja, conformada no sólo por una traditio directa de 36 manuscritos además de la edición moguntina de 1729, sino también por una traditio indirecta que no puede ser obviada, pues las partes I y II de la obra se reproducen literalmente en la Sententia definitiva sobre la ortodoxia luliana de 1419, obra reimpresa en multitud de ocasiones entre los siglos XVI-XVIII (a estudiar este fenómeno se dedican las pp. 95-105 del volumen).

Cada una de las obras editadas se acompaña de un aparato crítico dispuesto en dos niveles: en el primer nivel, se anotan los aspectos culturales más significativos del texto; en el segundo, se consignan las variantes transmitidas por la tradición manuscrita e impresa del mismo. Además, al final del volumen se incorporan índices (de citas bíblicas, de nombres y de títulos) que facilitan al lector la localización y consulta de los contenidos. Estamos, en definitiva, ante una obra que aúna rigor filológico y un profundo conocimiento de la materia abordada para ofrecernos, amén de unos textos pulcramente editados, las necesarias claves para entender las circunstancias de su composición y el mensaje que el autor quería transmitir con ellos. Sin duda, se trata de una muy valiosa adición al campo de los estudios lulianos.

JOSEP ANTONI AGUILAR ÁVILA

Universidad Católica de Valencia

\title{
JOSÉ HIGUERA RUBIO, Física y teología (Atomismo y movimiento en el Arte luliano). Madrid, Círculo Rojo, 2014, 326 pp., ISBN: 9788490508190
}

Queremos acercarnos aquí, en esta breve nota o recensión bibliográfica, a una, o mejor, dos piezas de interés en el marco de la galaxia luliana. Responden, por así decir, a trabajos preparatorios del Any Llull (2017) (700 aniversario de la muerte de R. Llull, uno de los pilares de la filosofía hispana), de emotivo recuerdo, que se ha celebrado en Baleares, Cataluña, incluso en Galicia, el Estado Español, Europa, Iberoamérica y América del Norte, con numerosos actos académicos, propuestas de investigación y monografías.

El profesor José Higuera Rubio no es precisamente un desconocido en el mundo del lulismo. Colaborador del Departamento de Filosofía III (Universidad Complutense de Madrid), donde imparte seminarios sobre las formas de transmisión de la tradición filosófica medieval, y del Institut d'Estudis Medievals (IEM) adscrito a la Universitat Autònoma de Barcelona (dirigido José Enrique Ruiz-Domènec y Alexander Fidora), al que está estrechamente ligada la monografía que citamos en segundo lugar. Es figura relevante en los medios académicos internacionales, en su vertiente investigadora, tanto en relación con la historia de la filosofía medieval como con el lulismo. Sobra decir que, en este último campo, ha llevado a cabo una empresa titánica: la fundación, junto con otros, pero con protagonismo propio, en 1999, del Instituto Brasileiro Raimundo Lúlio (São Paulo, Brasil) (IBFCRL), interconectado desde entonces con el mencionado IEM, con el Raimundus-Lullus-Institut de la Universidad de Friburgo, en el que ha desempeñado un papel editorial muy importante en los últimos años 
nuestros paisano muy querido Fernando Domínguez Reboiras, y, por supuesto, con la Société Internationale pour l'Étude de la Philosophie Médiévale (SIEMP), con sede en Lovaina, en la que coordina la «Sección Lulliana». Es editor para Brepols, precisamente, del volumen dedicado a la Sección Lulliana del Congreso SEIMP-Freising (2012). Hoy en día se encuentra incorporado al Gabinete de Filosofía Medieval y al Instituto de Filosofia, que dirigen J. Meirinhos y Paula Oliveira e Silva, en la Facultade de Letras de la Universidade de Porto (Portugal). Desde el 2012 no sólo coordina un grupo interdisciplinario dedicado al estudio de la obra filosófica luliana (Sección Lulliana- SIEMP-Commission of Latin Philosophy, ya mencionada), sino que, al tiempo, encabeza un proyecto de investigación, «The Reconstructión of 'Tree of Knowledge': Historical Contingencies and Textual Transformations», desde 2014 (GFM, IF, FLUP), y que, con seguridad, traerá importantes frutos, nunca mejor dicho, para la investigación medieval y luliana. A nivel metodológico, confiesa él mismo, aplica la perspectiva de los procesos de larga duración a las transformaciones conceptuales de la historia y filosofía de la ciencia, lo que explica ciertamente que gran parte de su investigación verse sobre historia de la lógica y de la ciencia, y la transmisión del lulismo a la temprana Modernidad.

Su obra Física y teología (Atomismo y movimiento en el Arte luliano) (2014) queda enmarcada en una línea de trabajo que el editor explica muy bien en la solapa del propio texto: «En la historiografía de la ciencia persiste una pujante distinción entre la física aristotélica de las causas y la física de la experimentación moderna, así como una especie de 'vacío' entre estos dos modelos. Este 'vacío' tiene su punto más fuerte en el lenguaje matemático usado para representar las variaciones cuantitativas y cualitativas del movimiento. No obstante, algunos autores medievales como Ramon Llull, que supuestamente trataban temas considerados extra-científicos, formularon las bases para que fuera posible la recepción del lenguaje de la física aristotélica y el lenguaje matemático. Esto implica una continuidad — gradual— entre el lenguaje de la física aristotélica y en el lenguaje matemático basada en dos grandes cambios: la aparición de una nueva perspectiva acerca de la cuantificación y las relaciones entre los objetos aplicadas al movimiento, y la discusión atomista acerca de las partes mínimas que componen la naturaleza». El autor aborda esta problemática con el rigor que se le supone, y con la claridad — cortesía del filósofo, según sentencia de D. José Ortega y Gasset, nuestro exiliado liberal en Portugal - necesaria, por lo general lejana de algunas ramas encriptófilas del lulismo. Es libro pensado para el lector especializado, para el especialista, pero que, de hecho, no echa para atrás a la persona curiosa por más lega que sea. Hagamos una cartografía del mismo. El libro de J. Higuera está dividida en 8 grandes capítulos y Conclusiones («El Arte luliano como semiofísica»), que pasamos a enumerar: Cap. 1, «Naturaleza y Arte», donde se analiza el Arte luliano y la transformación de la física aristotélica, las configuraciones del realismo geométrico luliano y, finalmente, la physica luliana; un Cap. 2, «La dinámica de lo divino», que toca aspectos varios, el proceso de retroalimentación filosofíateología, la filosofía sobrenatural, los principios filosóficos, el «medio» y el «flujo», la cantidad, y las cuestiones derivadas de las Sentencias y la condena de 1277; Cap. 3, «El lenguaje analítico en el Arte luliano», donde abordan otro buen conjunto de cuestiones, el De natura relationis, con la inversión luliana del estatus predicamental de las relaciones, la «rehabilitación» teológica de las relaciones, las especies de la relación y los principios relativos del Arte luliano, los correlativos (propiedades relacionales y libertad denominativa), simultaneidad y contrariedad en las relaciones, el lenguaje de los correlativos (una tradición factible), los correlativos (un lenguaje analítico para la dinámica del cambio: movimiento, mutación sustancial, generación y corrupción); Cap. 4, «La representación y estructura correlativo-geométrica de la combinación de los elementos», con precisiones sobre el «atomismo» luliano y la realidad de las partes simples de las cualidades elementales (lo discreto, lo continuo y lo contiguo), la composición geométrico-correlativa de los sujetos compuestos de elementos, las cualidades elementales como referencia física, significación correlativa de la mixtio elemental, la representación geométrica de la mixtio elemental; Cap. 5, «La estructura correlativo-geométrica de la naturaleza»; Cap. 6, «La nueva física: El movimiento, el tiempo, el lugar y el infinito», donde se habla de la vía del perfeccionamiento (composición y categorización del movimiento). La creación o «infusión» del movimiento en la naturaleza, el comportamiento físico de los atributos divinos, o la cualificación de las virtudes espirituales; Cap. 7, «Lugar y tiempo (ubi et quando)», donde se plantean 
una serie de problemas y temas, la duración y el instante (duratio et quando), la ontología del tiempo, la cuestión del «¿Cuándo?», sucesión y simultaneidad; Cap. 8, y último, «La nueva física y la disputa sobre la fuerza infinita», la interpretación de la física aristotélica, la causa primera, la potencia infinita, los catálogos de errores como género filosófico medieval y la polémica luliana. Hasta aquí la cartografía del texto de J. Higuera, que cuenta con la garantía del especialista, y que se adentra en novedosos confines de la gran galaxia del lulismo (física y atomismo).

Y, con todo, decir esto es decir poco, es quedarse en el «afuera» del texto. Es cierto que el pensamiento de Ramon Llull, en general, es un pensamiento ensimismado. No. No queremos decir con esto que no dialogue con próximos y lejanos. El texto de Higuera lo prueba. Ahí hay mucho de transacción en una y otra dirección. Dialoga con la tradición escolástica, con árabes y judíos, como siempre, sobre éste o cualquier otro tema. Pero quedarse aquí, tal vez, para los que creemos que caben muchas otras lecturas, casi infinitas, de su pensamiento, y de todo, comprendiendo el esfuerzo del autor en su monografía, nos sigue sabiendo el escrito a poco, o, dicho con mayor precisión, nos deja con ganas de más. No queremos agobiar a nuestro estudioso, claro. Pero, tal vez, para que la interpretación no quede definitivamente plegada al «ensimismamiento» de la obra estudiada, tal vez, decimos, fuera necesario un esfuerzo añadido, y en una segunda fase, y suponemos que J. Higuera Rubio podría hacerlo, pues competencias no le faltan y entusiasmo tampoco (si sus muchos compromisos y responsabilidades académicas y universitarias se lo permitiesen), estudiar, a propósito de estas dos materias que aborda (que terminan convergiendo, física y teología, atomismo y movimiento), el diálogo ad extra. Intentaré resumir el contenido de su trabajo: para el autor, el pensamiento de Llull es una teología de la relación y en una física de la relación («a trabe de ouro» del pensamiento luliano, empleando aquí una conocida expresión de un narrador gallego), a partir de la procesión o relación al infinito ad intra trinitario, hasta extenderlo (expandirlo) al conjunto de la realidad, ad extra, que, por cierto, no es una realidad o universo infinito, pues asume el repliegue de la potentia absoluta a la potentia ordinata, en apelación a la voluntad divina, narrada en el Génesis, en la pagina sacra, como podría plantear un contemporáneo suyo, como Juan Duns Scoto (1266-1308). Por lo cual, esta física sagrada, alternativa a la ptolemaico-aristotélico-tomista, mantiene un encuadre similar en muchos puntos a ésta: de cara a la modernidad, recaída en el «finitismo», primer obstáculo epistemológico, y, por otro lado, pese a la novedad, y al universo del dinamismo físico que evocan y registran las Artes, sigue presa — segundo obstáculo epistemológico — del corsé del «ejemplarismo» agustiniano-franciscano. Todo esto hay que tenerlo siempre presente y en cuenta.

Pero más arriba hablábamos de otra cosa: de la obligación que el especialista, el intérprete, tiene de avanzar todavía un paso más allá en el desciframiento de este diálogo, ahora cara afuera, en el tiempo y en el espacio, con otros autores y sobre estos mismos asuntos, a propósito de esta física de la relación y esta teología de la relación. Yo le propongo al autor, a J. Higuera, tres nuevos retos: $1^{\circ}$ ) el atomismo, tan vilipendiado en Occidente como el pirronismo, en la cultura árabe contemporánea a Llull, tuvo también un uso sacro, en el marco de la teología racional ash 'arita, donde, como nos explicaba E. Gilson, el ocasionalismo convergía con el atomismo, por ahí iría una primera vía de diálogo fuera del contenido ya en el texto y en el estudio; $2^{\circ}$ ) en segundo lugar, y causa cierto desconcierto, también en la contra-epistemología escéptico-pirrónica, neo-pirrónica y aún empírica, aquella que está regulada por la tabla de las contra-categorías aristotélicas configurada por los 10 tropos de Enesidemo (contra los sentidos) y los 5 modos de Agripa (contra la razón), se produce una inversión, pasando la categoría de ousía al final de la tabla (hasta propiamente desintegrarse) y la de «relación» a la cúspide de la misma: es la contra-categoría del «prós ti», $\pi \rho o ́ \varsigma$ $\tau \iota$ (Sexto Empírico, H.P., I, xiv-xv); de donde se deduce que, como era de esperar, la categoría de «relación» puede ir en direcciones contrarias, simultáneamente, contradictorias, tanto para asentar y asegurar una verdad de fe, cristiana o del credo que fuere, como para erosionar cualquier tentativa de garantizar un principio de verdad o certeza en el asunto mundano más trivial; creemos, por lo tanto, que una revisión de la temática desde esta otra perspectiva, ayudaría a completar lo planteado en nuestro libro; $3^{\circ}$ ) podríamos decir algo del Cardenal de Cusa, pero veamos que ocurre en otro autor, que le debe mucho a Llull y al Cardenal de Cusa (muy interesado en Llull), nos referimos al gran Nolano, Giordano Bruno de Nola, nacido en 1548, 
reo de Inquisición y quemado en la hoguera en 1600 en la plaza del Campo de las Flores de Roma - todavía su presencia se hace notar allí merced a la escultura de Ettore Ferrari (1845-1929) - por impiedad, materialismo y panteísmo; pues bien, sabemos que él supo combinar también las artes lulianas y el atomismo, pero no el lógico, o teológico, sino empírico y físico, el de raigambre y solera leucipo-democritea, epicúrea y lucreciana, como lo prueba, por referirme tan sólo a sus obras postreras, De lampade combinatoria Lulliana (1587) y la tríada De triplici minimo et mensura, De monade numero et figura y De innumerabilibus, immenso, et infigurabili (1591) (fue encarcelado en 1592), en ellas defiende un atomismo en el contexto de una ciencia laica y ajena a la pagina sacra, un universo infinito en el que la divinidad se manifiesta en la grandeza de la metamorfosis perpetua de la materia, natura est deus in rebus, una física no-sacra.

Son tres nuevos escenarios, tres propuestas-diálogos ad extra, por si el autor, el Profesor, Doctor e investigador J. Higuera Rubio, quisiese recorrerlos, pues el corpus luliano, por suerte, se halla siempre en expansión. Y, ya para finalizar, unas palabras tan sólo sobre otro libro, aparecido en diciembre de 2017, muy recientemente por lo tanto, casi salido del horno: En torno a Ramon Llull. Presencia y transmisión de su obra. Editores: Francisco José Díaz Marcilla y José Higuera Rubio. V. N. de Famalicão: Edições Húmus (Col. «Textos \& Estudios de Filosofía Medieval», núm. 11, dir. J. Meirinhos, Gabinete de Filosofia Medieval / Instituto de Filosofía, Facultade de Letras da Universidade de Porto, FLUP), 2017.

Textos recopilados por J. Higuera y el historiador Díaz Macilla, correspondientes a varios congresos sobre el pensamiento de Llull previos a la celebración del VII centenario de su muerte: $1^{\circ}$ ) por un lado, los estudios lulistas presentados al congreso de SIEMP en Palermo en el año 2007 (en el marco del Congreso Internacional presidido por el ya desaparecido y siempre llorado, lulista Musco), en las sección organizada por el gallego Fernando Domínguez Reboiras, así como en la misma sección, pero en el congreso de SIEMP en Fresing en el año 2012, coordinada por J. Higuera; y, $2^{\circ}$ ), por otro lado, dos encuentros, realizados al amparo de la Sección Luliana: «El nacimiento de la Universidad en Europa: Ramon Llull y las disputas universitarias» (Palma de Mallorca, 2013, organizado por Antoni Bordoy de la Universitat de les Illes Balears) y «Em torno de Raimundo Lulo» (organizado por Francisco José Díaz Marcilla, del Instituto de Estudos Medievais-Universidade Nova de Lisboa, en Lisboa, 2014).

Nos limitaremos tan sólo aquí a indicar el contenido del mismo. Además de una Introducción de los coordinadores, está dividido en dos amplias secciones, la primera titulada Pensamiento Luliano, recoge 4 trabajos, a saber, «Ramon Llull y la discusión en torno a la existencia del averroísmo latino (siglos XIXXX): el caso de la Declaratio Raimundi per modum dialogo edita», de Antoni Bordoy, «Ramon llull y la época. Génesis histórica del pensamiento luliano», de Gabriel Ensenyat Pujol, «Las subidas y bajadas de Ramon Llull, Antologías místicas y lógicas», de Walter Redmond, y, finalmente, de Constantin Teleanu, «Averroes et Averroiste christianus: deux adversaires de Raymond Lull à l'Université de Paris»; y, una segunda parte, titulada Historia del Lulismo, con las siguientes colaboraciones: «El lulismo europeo medieval: una aproximación», de F. J. Díaz Marcilla, «O amor divino n’ O libro do amigo e do amado: influencia do sufismo em Raimundo Lulo», de Natália Maria Lopes Nunes, «Ramon Llull y la concepción humanista del amor (El De amore de Alain de Varènes, un lulista 'menor' del círculo lefevriano)», del propio J. Higueras Rubio, «Una apostilla su Girolamo Cardano e il lullismo», de Carla Compagno y Rafael Ramis Barceló, «Raimundo Lulo e o Livro da Corte Imperial: um exemplar de literatura de polémica judaicoreligiosa medieval», de Alice Tavares, y, finalmente, de Alessandro Tessari y Alberto Pavanato, «1598: Annus Mirabilis». El cruce de lenguas sigue enriqueciendo el legado luliano. Sobre este último libro, de 196 páginas, sólo cabe decir que tiene todas las garantías de excelencia de cualquier producto, académico o de investigación, que salga o esté relacionado con la factoria del Gabinete de Filosofía Medieval de la FLUP. Solo cabe felicitarnos de ambas aportaciones al Any Llull (2017).

MARTÍN GONZÁLEZ FERNÁNDEZ Universidad de Santiago de Compostela 